\title{
Adhesive loose packings of small particles
}

\author{
Wenwei Liu ${ }^{1}$, Shuiqing $\mathrm{Li}^{1 *}$, Adrian Baule ${ }^{2}$, Hernán A. Makse ${ }^{3 \dagger}$ \\ ${ }^{1}$ Key Laboratory for Thermal Science and Power Engineering of Ministry of Education, \\ Department of Thermal Engineering, Tsinghua University, Beijing 100084, China \\ ${ }^{2}$ School of Mathematical Sciences, Queen Mary University of London, Mile End Road, London E1 4NS, UK \\ ${ }^{3}$ Levich Institute and Physics Department, City College of New York, New York 10031, USA
}

(Dated: October 12, 2018)

\begin{abstract}
We explore adhesive loose packings of dry small spherical particles of micrometer size using 3D discrete-element simulations with adhesive contact mechanics. A dimensionless adhesion parameter $(A d)$ successfully combines the effects of particle velocities, sizes and the work of adhesion, identifying a universal regime of adhesive packings for $A d>1$. The structural properties of the packings in this regime are well described by an ensemble approach based on a coarse-grained volume function that includes correlations between bulk and contact spheres. Our theoretical and numerical results predict: (i) An equation of state for adhesive loose packings that appears as a continuation from the frictionless random close packing (RCP) point in the jamming phase diagram; (ii) The existence of a maximal loose packing point at the coordination number $Z=2$ and packing fraction $\phi=1 / 2^{3}$. Our results highlight that adhesion leads to a universal packing regime at packing fractions much smaller than the random loose packing, which can be described within a statistical mechanical framework. We present a general phase diagram of jammed matter comprising frictionless, frictional, adhesive as well as non-spherical particles, providing a classification of packings in terms of their continuation from the spherical frictionless RCP.
\end{abstract}

Jammed particle packings have been studied to understand the microstructure and bulk properties of liquids, glasses and crystals 1, 2] and frictional granular materials [3, 4. Two packing limits have been identified for disordered uniform spheres: The random close packing (RCP) and random loose packing (RLP) limits [1, 511. The upper RCP limit is reproduced for frictionless spheres at volume fractions $\phi \approx 0.64$ and has been associated with a freezing point of a 1st order phase transition [12 15], among other interpretations [2, 16]. In the presence of friction, packings reach lower volume fraction up to the RLP limit $\phi_{\mathrm{RLP}} \approx 0.55$ for mechanically stable packings [6, 8, 11]. However, most packings of dry small micrometer-sized particles in nature are not only subject to friction, but also adhesion forces. In fact, van der Waals forces generally dominate interactions between particles with diameters of around $10 \mu \mathrm{m}$ or smaller. In this case, the adhesive forces begin to overcome the gravitational and elastic contact forces acting on the particles and change macroscopic structural properties [17, 18.

Despite the ubiquity of adhesive particle packings in almost all areas of engineering, biology, agriculture and physical sciences [18 21, these packings have so far not been systematically investigated. The multi-coupling of adhesion, elastic contact forces and friction within the short-range particle-particle interaction zone and their further couplings with fluid forces (e.g., buoyancy, drag and lubrication) across long-range scales make it highly difficult to single out the effect of the adhesion forces

\footnotetext{
*lishuiqing@tsinghua.edu.cn

${ }^{\dagger}$ hmakse@lev.ccny.cuny.edu
}

alone. Previous studies have found packing fractions of adhesive microparticles in a wide range of $\phi=0.07-0.33$ for both uncompressed and compressed samples using a random ballistic deposition technique 22. A similarly broad range of $\phi=0.23-0.41$ was found for particles with diameter of $7.8-19.1 \mu \mathrm{m}$ using fluidized bed techniques 23 .

In this letter, a prototypical packing system is introduced for the simulation of random loose packings of soft-sphere, non-Brownian, adhesive particles with a discrete element method (DEM). Here, the fluid effect is filtered out by assuming the gravitational sediment under a vacuum condition. Most importantly, the gravitational effect can be neglected when the system satisfies $F_{r}=U_{0} / \sqrt{g H}>>1$, where $F_{r}$ is the Froude number (ratio of inertia to gravity), $H$ the characteristic height of the deposition control volume and $U_{0}$ the initial particle velocity at the upper inlet boundary. For all runs in the numerical simulations, we ensure that gravitational effects with respect to initial particle inertia are less than $3 \%$. Therefore, the adhesive packings simply arise due to the competition between the particle inertia and particleparticle interactions (e.g., adhesion, elasticity and frictions).

In a novel DEM framework specifically developed for adhesive grains [17, 18, both the transitional and rotational motions of each particle in the system are considered on the basis of Newton's second law (see Supplementary Information, Section I). The adhesive contact forces $F_{A}$ include three terms, the normal adhesive contact force $F_{\text {ne }}$, the normal damping force $F_{n d}$ validated by classic particle-surface impact experiments 17, 18, and the tangential force due to the sliding friction. A JKR (Johnson-Kendall-Roberts) model is employed to 
account for $F_{\text {ne }}$ between the relatively compliant microparticles, implying the length scale of elastic deformation is large compared to the length scale of the adhesive force (with the particles' Tabor parameter larger than unity) [18. The dissipation terms, including the sliding, twisting and rolling frictions in the presence of adhesion, are all approximated by a linear spring-dashpot-slider model with model parameters given in 24. The slider considerations mean that the sliding, twisting and rolling resistances all reach critical values, $F_{s, \text { crit }}, M_{t, \text { crit }}$ and $M_{r, \text { crit }}$, as three related displacements exceed the certain limits. For displacements larger than those limits, the resistances stay constant and the particles start to slide or spin. The critical limits in presence of adhesion are given in the following equations [18, 19, 25],

$$
\begin{aligned}
F_{s, \text { crit }} & =\mu_{f}\left|F_{\mathrm{ne}}+2 F_{C}\right| \\
M_{t, \text { crit }} & =3 \pi a F_{s, \text { crit }} / 16 \\
M_{r, \text { crit }} & =-4 F_{C}\left(a / a_{0}\right)^{3 / 2} \Theta_{\text {crit }} R
\end{aligned}
$$

where $\mu_{f}$ is the friction coefficient, $\Theta_{\text {crit }}$ is the critical angle for the relative rolling of two particles, and $F_{C}$ is the critical pull-off force expressed by the work of adhesion (twice the surface energy, $w=2 \gamma$ ): $F_{C}=1.5 \pi w R$. Here, $R$ is the effective radius between two contacting particles $\left(1 / R=1 / r_{p_{1}}+1 / r_{p_{2}}\right), a$ is the radius of the contact area with $a_{0}$ at equilibrium in the JKR model. The values or ranges of $\mu_{f}, \Theta_{\text {crit }}$ and $F_{C}$ are selected according to the data from atomic force microscopy measurements [25]27.

The adhesive DEM simulation starts with the random free falling of 1,000 spheres with an initial velocity $U_{0}$ at a height $H$ (see the Supplementary Information, Section II, for detailed physical and geometrical parameters). The horizontal plane for particle deposition has two equal edges of length $L$, with periodic boundary conditions along the two horizontal directions. Here we focus on uniformly sized particles, with particle radius ranging from $r_{p}=1$ to $50 \mu \mathrm{m}$. A sensitivity analysis shows that the difference in $\phi_{\mathrm{RLP}}$ between the cases $L=20 r_{p}$ and $L=40 r_{p}$ is negligible, indicating the $L=20 r_{p}$ is large enough to reproduce bulk properties. Then, we set $L=20 r_{p}$. The work of adhesion, e.g., for silica microspheres is reported at $20-30 \mathrm{~mJ} / \mathrm{m}^{2}$ [18, 25]. Setting $w=30 \mathrm{~mJ} / \mathrm{m}^{2}$, the simulations indicate that both particle deposition velocities and particle sizes significantly affect packing structures. As shown in Fig. 1 (more details in supplementary Fig. S2), either large velocity and small size, or small velocity and large size can produce a relatively dense packing. A dimensionless adhesion parameter $A d=w /\left(2 \rho_{p} U_{0}^{2} r_{p}\right)$, defined as the ratio between interparticle adhesion and particle inertia by $\mathrm{Li}$ and Marshall [28, can be used to quantify the combined effect of velocity and size. Figure 2 shows the variation of packing volume fraction as a function of $A d$ for packings with $r_{p}=1,5,10,50 \mu m$ with $w$ ranging from $5-30 m J / m^{2}$. In

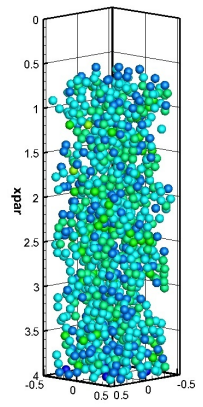

(a)

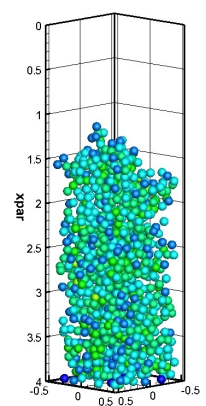

(d)

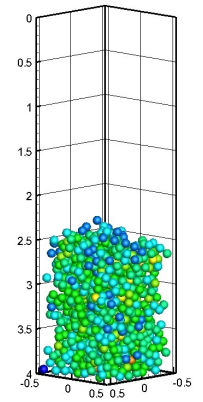

(b)

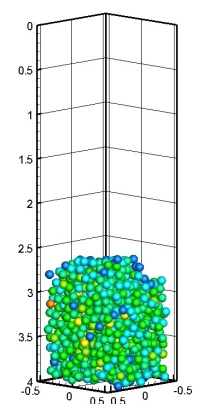

(e)

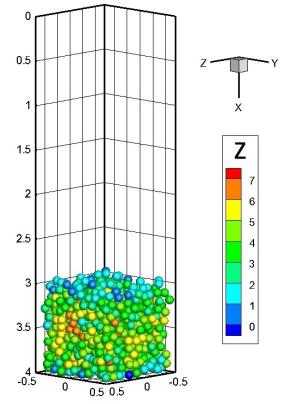

(c)

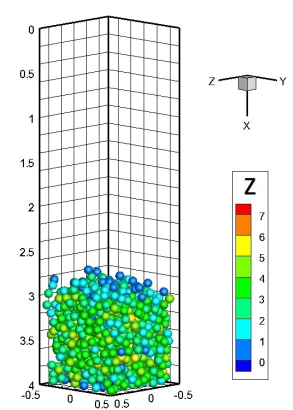

(f)
FIG. 1: (Colors online) Typical packing structure: different colors represent different coordination numbers $Z$. (a)(b)(c) stand for $U_{0}=0.5,2$ and $6 \mathrm{~m} / \mathrm{s}$, respectively with particle radius $r_{p}=1 \mu \mathrm{m}$ and work of adhesion $w=30 \mathrm{~mJ} / \mathrm{m}^{2}$. (d)(e)(f) stand for $r_{p}=1,5$ and $10 \mu \mathrm{m}$, respectively with $U_{0}=1 \mathrm{~m} / \mathrm{s}$ and $w=30 \mathrm{~mJ} / \mathrm{m}^{2}$.

the case of $A d<1$, the data points are scattered between RLP (0.55) and RCP (0.64), since particle inertia dominates the adhesion and frictions. However, when $A d>1$, we obtain an adhesion-controlled regime, in which the volume fraction decreases exponentially with increasing $A d$, becoming linear at large $A d \sim 10$. The lowest packing density achieved is at $\phi=0.154$ when $A d$ is as high as 96 , which agrees well with the data from a random ballistic deposition experiment [22].

In addition to $\phi$, a reproducible observable of the packing is the coordination number $Z$, which denotes the average number of contacts of a sphere in the packing. The isostatic conjecture predicts the upper and lower bounds of $Z=2 d_{f}$ and $Z=d_{f}+1$ for frictionless and infinitely rough hard-spheres, respectively, with $d_{f}=3$ degrees of freedom. In Fig. 2 (inset) we see that for $A d<1$ the packings lie indeed within the isostatic limits reaching the infinitely rough value $Z=4$ at $A d \approx 1$. This indicates that weak adhesion has a similar effect on the packing as strong friction. For $A d>1$ the adhesive packings fall on a unique curve, analogous to the $\phi$ dependence. The lowest $Z$ reached in our simulations is $Z=2.25$. Combining our results for $\phi$ and $Z$ thus highlights a universal adhesive regime characterized by the dimensionless parameter 


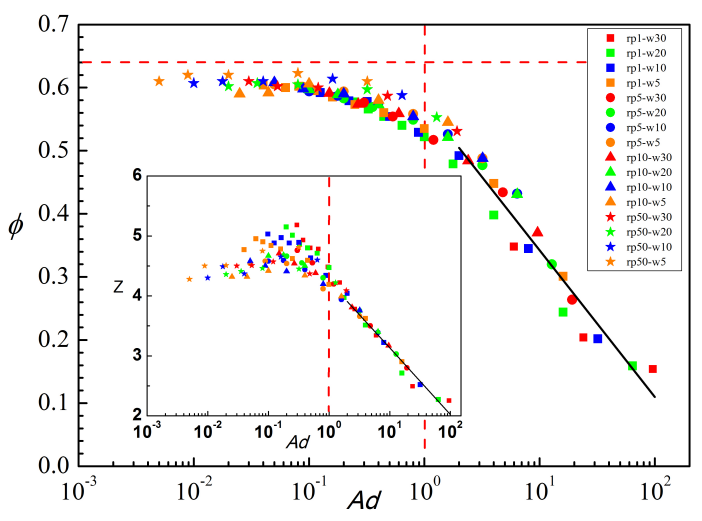

FIG. 2: (Colors online) Semi-log plot of the packing volume fraction as a function of adhesion parameter. The horizontal red dash line indicates the limitation of $\phi_{R C P}=0.64$ and the vertical one is the separation of $A d=1$. The inset shows the variation of the coordination number $Z$ with $A d$.

$A d$. The resulting curve in the $Z-\phi$ plane, parametrized by $A d$, can be considered as an equation of state of packings dominated by adhesion (see Fig. 3).

We now derive an analytical representation of the adhesive equation of state. To this end we introduce a statistical mechanical framework for particles with adhesive interactions in the spirit of Edwards' ensemble approach [10, 29, 30]. We start with the Voronoi volume $W_{i}$ of a reference particle $i$, which provides a tessellation of the total volume of the packing: $V=\sum_{i=1}^{N} W_{i}$. The key step is to use a statistical mechanical description, where we consider the average Voronoi volume $\bar{W}=\left\langle W_{i}\right\rangle$. This implies that $V=N \bar{W}$ and the packing fraction follows as $\phi=V_{0} / \bar{W}$. Here, $V_{0}$ is the volume of a sphere with radius $r_{p}$ in the packing. In turn, $\bar{W}$ can be expressed exactly in terms of the pdf $p(c, Z)$ for finding the boundary of the Voronoi volume at a distance $c$ from the sphere centre for a given $z$. We have [10, 29]

$$
\bar{W}=V_{0}+4 \pi \int_{r_{p}}^{\infty} \mathrm{d} c c^{2} P(c, Z),
$$

where $P(c, Z)$ is the $\mathrm{CDF} ; p(c, Z)=-\frac{\mathrm{d}}{\mathrm{dc}} P(c, Z)$. For $P(c, Z)$ one can derive a Boltzmann-like form using a factorization assumption of the multi-particle correlation function into pair correlations [30]

$$
P(c, Z)=\exp \left\{-\rho \int_{\Omega(c)} \mathrm{d} \mathbf{r} g_{2}(\mathbf{r}, Z)\right\} .
$$

Here, $\rho=N / V=1 / \bar{W}$ is the number density and $g_{2}(\mathbf{r}, Z)$ the pair correlation function of two spheres separated by $\mathbf{r}$. The volume $\Omega(c)$ is an excluded volume for the $N-1$ spheres outside of the reference sphere, since otherwise they would contribute a Voronoi boundary smaller than $c$. Typical models for $g_{2}$ consist of a step function plus a delta-peak to model the contribution of bulk and contacting particles (with a given coordination number $Z$ ), respectively [30, 31]: $g_{2}(\mathbf{r}, Z)=$ $\Theta\left(r-2 r_{p}\right)+\frac{Z}{\rho \lambda} \delta\left(r-2 r_{p}\right)$, where $\lambda$ is an appropriate constant. In order to describe the effect of correlations due to adhesion, we assume a gap of width $b(Z)$ that models the increased porosity at a given $Z$ compared with adhesion-less packings. This suggests to express $g_{2}$ as

$$
g_{2}(\mathbf{r}, Z)=\Theta\left(r-\left(2 r_{p}+b(Z)\right)\right)+\frac{Z}{\rho \lambda} \delta\left(r-2 r_{p}\right) .
$$

Clearly, $b(Z)$ is a smoothly decreasing function, so that we can assume, e.g., the simple parametric form $b(Z)=$ $c_{1}+c_{2} e^{-c_{3} Z}$. There are then two natural boundary conditions that constrain $\left(c_{1}, c_{2}, c_{3}\right)$, the three parameters in the theory: (i) At the isostatic limit $Z=6$, we expect to recover the frictionless RCP value, since this value of $Z$ represents a maximally dense disordered packing of spheres. We obtain from Eqs. (4) - (6) indeed the prediction of Ref. [10] for RCP at $\phi=\sqrt{3} /(1+\sqrt{3})=0.634$ provided we have $b(6)=0$ and $\lambda$ assumes the value $\lambda=$ $16 \pi r_{p}^{2} / \sqrt{3}$. Moreover, we need to account for low dimensional corrections due to the hard-core excluded volume of the reference sphere, such that $\rho \rightarrow \bar{\rho}=1 /\left(\bar{W}-V_{0}\right)$, where $V_{0}$ is the volume of a sphere with radius $r_{p}[30$. The constraint (i) thus fixes $\rho$ and $\lambda$, as well as one of the parameters in $b(Z)$, say $c_{1}$. (ii) In addition, we conjecture the existence of a maximally loose packing (MLP) at $Z=2$ and $\phi=1 / 2^{3}$ which yields $b(2)=1.47$ and fixes a second parameter, $c_{2}$. This is motivated by the fact that $\phi=1 / 2^{d}$ is the well-known lower bound of saturated sphere packings in $d$ dimensions [31. Moreover, $Z=2$ is the lowest possible value for a physical packing: If $Z<2$ there are more spheres with a single contact (i.e., dimers) than with three or more contacts.

Solving Eqs. (4)-(6) numerically for $\bar{W}$ (and thus $\phi$ ) with the functional form of $b(Z)$ leads to a family of curves with a single free parameter, $c_{3}$. Fitting this parameter to the available data yields $c_{3}=1$. We then obtain a unique equation of state $\phi(Z)$ for adhesive packings as shown in Fig. 3 which agrees remarkably well with the simulation data. Furthermore, the adhesive $\phi(Z)$ equation of state appears as a continuation of the frictionless RCP at $(Z, \phi)=(6,0.634)$. For large $A d$ values the MLP point at $(2,0.125)$ is indeed approached in the $Z$ - $\phi$ plane. A comparison of the theoretically obtained $P(c, Z)$ with simulation data is shown in the inset of Fig. 3. We observe that the agreement is good for small $c$ values throughout the range of adhesive packings. Since this range of $c$ values provides the dominant contribution to $\bar{W}$ in Eq. (4), we conclude that our phenomenological approach captures well the microstructure of adhesive packings in the first coordination shell, which provides a 


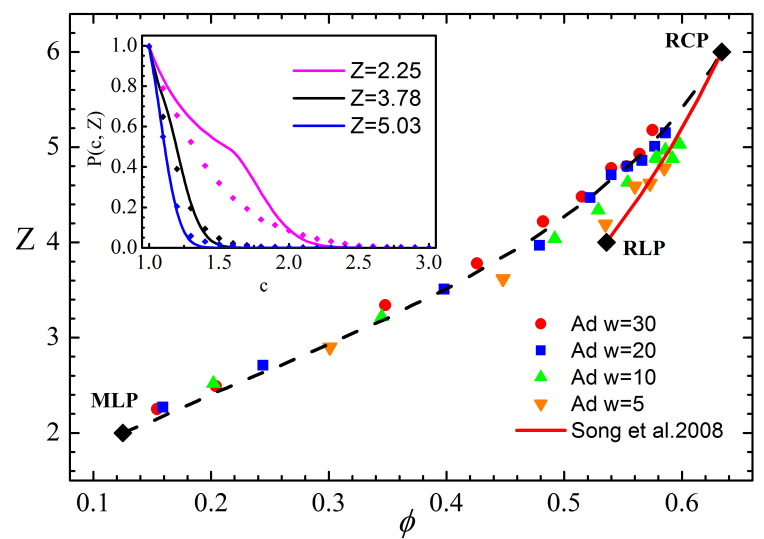

FIG. 3: (Colors online) Equation of state for adhesive packings: Simulation data and theoretical prediction from Eqs. (4)-(6) with a single fitting parameter (black dashed line). The equation of state for frictional sphere packings from Ref. 10] is indicated. Inset: Comparison of the CDF $\mathrm{P}(\mathrm{c}, \mathrm{Z})$ with simulation data (dots).

good first moment from $P(c, Z)$ as compared with simulations.

Including previous results from Refs. [10, 14, 29] in the $Z$ - $\phi$ plane leads to a phase diagram of packings of frictionless, frictional, and adhesive spheres, as well as non-spherical particles (see Fig. 4). The collection of these results highlights the prominent role of the frictionless RCP point in the phase-diagram, which appears as a focal point of the different packing classes. We observe in particular that the equation of state of disordered non-spherical packings is essentially smoothly continued at RCP into either the adhesive branch or the frictional branch. By contrast, the coexistence line from $\mathrm{RCP}$ to the melting point of crystalline packings, conjectured in Ref. 14, does not connect smoothly to any of these branches. The precise meaning of this crucial distinction is not entirely clear to us. It suggests that particle deformation (which parameterizes the non-spherical branch) is a "natural" way to increase packing densities in disordered arrangements. On the other hand, introducing order is a more drastic modification, similar to a distinction between discontinuous 1st and continuous higher-order phase transitions.

In summary, we have identified a universal packing regime of adhesive small particles across 1 to $10^{2}$ microns, using both DEM simulations and a statistical mechanical framework. We have shown that an equation of state for adhesive loose packings can be derived connecting the frictionless RCP with a conjectured universal MLP point in the phase diagram. The picture that emerges is that different classes of disordered packings are connected smoothly via RCP, while partially ordered phases are not. Clearly, further investigations are needed to understand

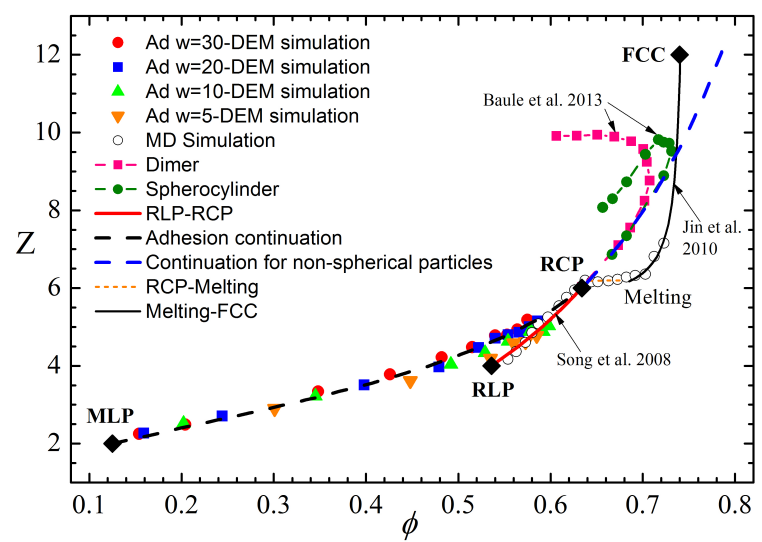

FIG. 4: (Colors online) The phase diagram of packings of different classes of monodisperse particles including the adhesive packings. The equation of state for non-spherical particles is smoothly continued at RCP into either the adhesive or the frictional branch.

the nature of packings in the vicinity of RCP, e.g., by probing the rheological properties of these packings close to jamming or by considering non-spherical adhesive particles. The MLP also deserves further attention. Open questions concern, e.g., the response of these packings to local perturbations or shear stresses. It would be highly interesting to find out whether a MLP is indeed observed in a real physical system.

The research is supported by the National Natural Science Funds of China (No.50976058), the National Key Basic Research and Development Program (No.2013CB228506), the basic-science funds from China Academy of Space Technology, NSF-CMMT and DOE Geosciences Division. We acknowledge M. Denn, J. Morris, C. S. O'Hern, E. Brown for helpful discussions. Thanks are due to J. Marshall, Q. Yao, G. Q. Liu, M. M. Yang and S. Chen for collaborations. AB acknowledges funding under EPSRC grant EP/L020955/1.

[1] J. D. Bernal, Nature (London) 183, 141 (1959).

[2] G. Parisi and F. Zamponi, Rev. Mod. Phys. 82, 789 (2010).

[3] A. Coniglio, A. Fiero, H. J. Herrmann, M. Nicodemi eds. Unifying Concepts in Granular Media and Glasses (Elsevier, Amsterdam, 2004).

[4] B. Andreotti, Y. Forterre, and O. Pouliquen, Granular Media between fluid and solid, p59-70 (Cambridge University Press, Cambridge, 2013).

[5] G. D. Scott, Nature 188, 908 (1960).

[6] G. Y. Onoda, and E. G. Liniger, Phys. Rev. Lett. 64, 2727 (1990).

[7] M. P. Ciamarra and A. Coniglio, Phys. Rev. Lett. 101, 128001 (2008).

[8] M. Jerkins, M. Schröter, H. L. Swinney, T. J. Senden, 
M. Saadatfar, and T. Aste, Phys. Rev. Lett. 101, 018301 (2008).

[9] K. J. Dong, R. Y. Yang, R. P. Zou, and A. B. Yu, Phys. Rev. Lett. 96, 145505 (2006).

[10] C. Song, P. Wang, and H. A. Makse, Nature 453, 629 (2008).

[11] G. R. Farrell, K. M. Martini, and N. Menon, Soft Matter 6, 2925 (2010).

[12] A. V. Anikeenko and N. N. Medvedev, Phys. Rev. Lett. 98, 235504 (2007).

[13] B. A. Klumov, S. A. Khrapak, and G. E. Morfill, Phys. Rev. B 83, 184105 (2011).

[14] Y. Jin and H. A. Makse, Physica A 389, 5362 (2010).

[15] A. Panaitescu, K. A. Reddy, and A. Kudrolli, Phys. Rev. Lett. 108, 108001 (2012).

[16] S. Torquato and F. H. Stillinger, Rev. Mod. Phys. 82, 2633 (2010).

[17] S. Q. Li, J. S. Marshall, G. Q. Liu, and Q. Yao, Prog. Energy Combust. Sci. 37, 633 (2011).

[18] J. S. Marshall and S. Q. Li, Adhesive particle flow: a discrete-element approach, p2, p81 (Cambridge University Press, Cambridge, 2014).

[19] C. Dominik, and A. G. G. M. Tielens, Astrophys. J. 480, 647 (1997).

[20] J. Blum, and R. Schräpler, Phys. Rev. Lett. 93, 115503
(2004).

[21] K. M. Kinch, J. Sohl-Dickstein, J. F. Bell III, J. R. Johnson, W. Goetz, and G. A. Landis, J. Geophys. Res. 112, E06S03 (2006).

[22] J. Blum, and R. Schräpler, B. J. R. Davidsson, and J. M. Trigo-Rodríguez, Astrophys. J. 652, 1768 (2006).

[23] J. M. Valverde, M. A. S. Quintanilla, and A. Castellanos, Phys. Rev. Lett. 92, 258303 (2004).

[24] M. M. Yang, S. Q. Li, and Q. Yao, Powder Technol. 248, $44(2013)$

[25] L.O. Heim, J. Blum, M. Preuss, and H. J. Butt, Phys. Rev. Lett. 83, 3328 (1999).

[26] R. Jones, H. M. Pollock, D. Geldart, A. Verlinden-Luts, Ultramicroscopy 100, 59 (2004).

[27] B. Siemer and M. Sitti, J. Adhesion Sci. Technology 22, 481 (2008).

[28] S. Q. Li and J. S. Marshall. J. Aerosol Science 38, 1031 (2007).

[29] A. Baule, R. Mari, L. Bo, L. Portal, H. A. Makse, Nature Commun. 4, 2194 (2013).

[30] Y. Jin, P. Charbonneau, S. Meyer, C. Song, and F. Zamponi, Phys. Rev. E 82, 051126 (2010).

[31] S. Torquato and F. H. Stillinger, Experimental Mathematics 15, 307 (2006). 